\title{
TV/Series
}

$7 \mid 2015$

Le Pilote et la chute

\section{«The show must go on » ou comment ralentir la chute}

Les dessous de la télévision dans Sports night, Studio 60 et The Newsroom

\section{Laure Depretto}

\section{(2) OpenEdition}

Journals

Édition électronique

URL : http://journals.openedition.org/tvseries/282

DOI : 10.4000/tvseries.282

ISSN : 2266-0909

Éditeur

GRIC - Groupe de recherche Identités et Cultures

Référence électronique

Laure Depretto, « «The show must go on » ou comment ralentir la chute», TV/Series [En ligne], 7| 2015, mis en ligne le 01 juin 2015, consulté le 22 avril 2019. URL : http://journals.openedition.org/ tvseries/282 ; DOI : 10.4000/tvseries.282

\section{(c) (i) (9)}

TV/Series est mis à disposition selon les termes de la licence Creative Commons Attribution - Pas d'Utilisation Commerciale - Pas de Modification 4.0 International. 


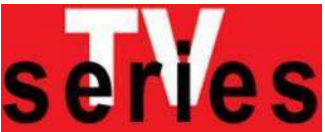

«The show must go on » ou comment ralentir la chute. Les dessous de la télévision dans Sports night, Studio 60 et The Newsroom

Laure DEPRETTO

Cet article analyse la capacité à durer de trois des quatre séries créées par Aaron Sorkin, Sports Night, Studio 60 on the Sunset Strip et The Newsroom, qui ont pour sujet les coulisses de la télévision. En optant pour un propos avant tout descriptif (faire découvrir les dessous et surtout les contraintes d'un programme télévisé), elles se privent d'emblée d'un suspense à long terme au profit d'une tension à courte portée (les personnages parviendront-ils à boucler leur show à temps ?) On étudie d'abord les trois pilotes programmatiques qui amorcent un même arc narratif (la tension entre l'ambition d'excellence et les impératifs économiques qui entravent les personnages dans l'accomplissement d'une émission de qualité), puis la manière dont chacune de ces séries organise la suite, ralentissant ou préparant sa chute. Ce faisant, on se propose d'examiner les rapports que ces séries entretiennent avec le feuilleton, le cycle, ainsi que les stratégies narratives qu'elles déploient, telles que l'insertion d'intrigues à suspense, pour se relancer et finir sans en finir.

This article analyses the three behind-the-scenes shows created by Aaron Sorkin, Sports Night, Studio 60 on the Sunset Strip and The Newsroom, especially their ability to last. By adopting a descriptive angle (how does a TV show work? what are its economic constraints?), they have adopted a short-range tension instead of a long-term suspense (will the characters complete their show in time?). First we will study the three pilots which are launching an identical story arc (i.e., the tension between quality and profit), then the narrative techniques the three series use to slow down or accelerate the ending. By doing so, we will examine the serial and cyclic nature of these series as well as the various strategies enabling them to last or start anew, including the insertion of sideways suspense stories.

$\mathrm{C}$

et article porte sur trois séries centrées sur les coulisses d'un show télévisé : Sports night (ABC, 1998-2000), Studio 60 on the Sunset Strip (NBC, 2006-2007), The Newsroom (HBO, 2012-2014). Elles ont d'abord comme point commun leur créateur: Aaron Sorkin. S'il est assez habituel en littérature de regrouper des œuvres à partir de leur auteur, le réflexe est sans doute moins naturel dans un art qui suppose des équipes de scénaristes et qui implique un travail collectif. Mais précisément, Aaron Sorkin est l'un des rares à s'être fait un nom dans l'univers des séries. Il est surtout connu pour sa série au long cours The West Wing (NBC, 1999-2006). En 2010, on entendit de nouveau beaucoup parler de lui comme scénariste du film de David Fincher, The Social Network ${ }^{1}$. Un style -

${ }^{1}$ C'est Sorkin qui a obtenu en 2011 l'Oscar du meilleur scénario adapté pour son adaptation du roman de Ben Mezrich, The Accidental Billionaires: The Founding Of Facebook, A Tale 
très vite baptisé, en bonne ou en mauvaise part, sorkinian, sorkinesque - a été identifié dans les types de personnages qu'il invente et dans son écriture des dialogues. Ses personnages semblent les héritiers directs de ceux des "screwball comedies" des années 1930 et 1940, des films de Capra, et des héroïnes de ce que Stanley Cavell a rassemblé dans le genre de la comédie du remariage ${ }^{2}$. Les séries de Sorkin sont fondées sur des dialogues agonistiques. Pour mettre en scène le dynamisme de ces échanges, Sorkin, en collaboration avec son réalisateur Thomas Schlamme, a promu une technique qui est devenue sa signature, son label : le walk and talk. Les personnages parlent en marchant, ce qui dynamise la scène, évite le traditionnel champ-contrechamp et permet de faire découvrir l'environnement des personnages en même temps que ces derniers exposent la situation. La virtuosité des dialogues, des femmes à forte personnalité, des idéalistes et des utopistes, une intelligence et une culture infinie, un humour slapstick : autant de traits sur lesquels la critique a pu ironiser, jugeant les personnages sorkiniens irréalistes 3 .

Les trois séries qui m'intéressent ici partagent aussi, au sein des créations de Sorkin, un même sort : elles ont, d'une manière ou d'une autre, été éclipsées par sa grande œuvre, The West Wing qui, à bien des égards, a servi et sert encore d'étalon-mesure. Que ce soit avant ou après, chacune d'elles a souffert de la comparaison. Sports Night a été perçu comme un galop d'essai, tandis que Studio 60 , série post-West Wing était attendue avec tant de curiosité et d'impatience qu'elle ne pouvait que décevoir. Quant à The Newsroom, on a beaucoup dit que Sorkin essayait de refaire avec la télévision ce qu'il avait fait avec la politique. Ce qui était reçu comme un coup de génie est apparu comme une recette, une formule répétitive.

Comme The West Wing, ces séries sont fondées sur un point de départ identique: montrer les coulisses d'un environnement professionnel fascinant, la façon dont travaillent des personnages publics qui exercent une influence sur nos vies quotidiennes et individuelles. L'ambition affichée par Sorkin était de contribuer à réenchanter et à réidéaliser des univers perçus comme cyniques et immoraux. Dans un entretien, Sorkin indique que son mode opératoire

of Sex, Money, Genius, and Betrayal, New York, Doubleday, 2009 (disponible en français, trad. Lucie Delplanque, La Revanche d'un solitaire : la véritable histoire du fondateur de Facebook, Paris, M. Milo, 2009).

2 Stanley Cavell, Pursuits of Happiness. The Hollywood Comedy of Remarriage, Cambridge, coll. "Harvard Film Studies», Harvard University Press, 1981; Kirstin Ringelberg, " His Girl Friday (and Every Day) : Brilliant Women Put to Poor Use » in Considering Aaron Sorkin: Essays on the Politics, Poetics ans Sleight of Hand in the Films and Television Series, éd. Thomas Fahy, Jefferson/Londres, McFarland and co, 2005, p. 91-100.

3 Voir, par exemple, Graeme McMillan, « Revisiting The West Wing: A Stirring, Comforting Fantasy », Time, 19 avril 2013, http://entertainment.time.com/2013/04/19/revisiting-thewest-wing-a-stirring-comforting-fantasy/ (consulté le 15 juin 2015). 
est toujours peu ou prou le même : choisir une institution dont on ignore tout, Sorkin compris (l'armée dans A Few Good Men [réal. Rob Reiner, 1992], la Maison Blanche dans The West Wing, le journalisme dans The Newsroom) et l'incarner dans des personnages pour l'humaniser 4 .

Plus spécifiquement encore, Sports Night, Studio 60 et The Newsroom sont ce qu'on appelle des séries behind the scenes ou encore des backstage shows (voir, en annexe, le tableau récapitulatif). Sports night est le nom d'une émission sportive quotidienne présentée par deux anchormen, Casey McCall (Peter Krause, qui sera plus tard le Nate de Six Feet Under [HBO, 2001-2005]) et Dan Rydell (Josh Charles, futur Will Gardner dans The Good Wife [CBS, 2009-]), sur une chaîne câblée qui a du mal à faire sa place, devancée par Fox et CNN. Studio 60 est une émission de divertissement où se succèdent des sketchs satiriques, diffusée en deuxième partie de soirée, écrite par une équipe et jouée par une poignée d'acteurs, constamment menacée par la chaîne, pour des raisons de censure ou d'audiences insuffisantes. Riche d'une longue histoire télévisuelle, le show a longtemps été dirigé par Wes Mendel, renvoyé dans l'épisode-pilote après avoir fait en direct une diatribe contre la télévision. Dès l'épisode 1, il est remplacé par ses deux fils spirituels, Daniel Tripp (Bradley Whitford, qui jouait déjà Josh dans The West Wing) et Matthew Albie (Matthew Perry de Friends). The Newsroom représente la fabrique d'un journal télévisé News Night, présenté par un anchorman vedette, Will McAcvoy (Jeff Daniels) et produit par une équipe de journalistes intègres emmenée par Mackenzie MacHale (Emily Mortimer).

Ce choix de montrer comment on travaille à la télévision suppose de s'inspirer du réel, en prenant même certains présentateurs et animateurs pour modèles. Si on le souhaite, ces séries peuvent êtres interprétées comme des séries à clés. Mais la chose est beaucoup plus difficile pour un spectateur français qui ne connaît pas les présentateurs en question et ne peut donc jouer au jeu de la reconnaissance. Cette question ne sera pas abordée ici, mais elle est d'importance : elle permet, me semble-t-il, d'expliquer la différence de réception de ces séries de chaque côté de l'Atlantique. Mon hypothèse est en effet que la critique française est plus dithyrambique que la critique américaine en partie parce que ce qui est dépeint et montré dans ces séries est plus exotique ici qu'aux États-Unis et que le sentiment de déjà-vu, déjà-connu y est moins fort. Parce que nous n'avons pas la même télévision, les mêmes mécanismes de production, ni le même système de diffusion, les trois séries sont beaucoup plus dépaysantes pour nous que pour un spectateur américain.

4 « Now with Alex Wagner », 28 juin 2012, MSNBC, http://www.msnbc.com/now-withalex-wagner/watch/aaron-sorkin-covers-the-newsroom-44421187653 (consulté le 15 juin 2015). 
Pour terminer cette présentation et pour en venir - enfin - à la question du début et de la fin, il convient encore de donner quelques indications sur le destin de chacune des trois séries. La fin de Sports Night, après deux saisons de vingt-deux épisodes chacune, relève d'une décision d'auteur : déjà engagé dans l'écriture et la promotion de The West Wing, Sorkin n'a pas souhaité mener les deux séries de front, alors que d'autres chaînes proposaient son rachat, dont HBO, USA et Showtime. Attendue avec impatience et lancée avec fracas, Studio 60 a échoué à se prolonger au-delà d'une saison alors que dans le même temps, la chaîne reconduisait 30 Rock (NBC, 2006-2013) de Tina Fey5. Les fans inconditionnels ont défendu l'idée que cette série trop exigeante avait été mal comprise; ses détracteurs que Sorkin s'était égaré dans un genre qui n'était pas le sien - il mettait trop de drame dans une comédie ${ }^{6}$. La série serait morte de son esprit de sérieux. Quant à la dernière, The Newsroom, au moment où nous écrivons ces lignes, elle est encore en cours : après deux saisons - 19 épisodes en tout - diffusés sur HBO, la chaîne a annoncé une troisième (et sans doute dernière) saison pour l'automne $2014^{7}$.

Face à des séries qui n'ont tenu qu'une ou deux saisons, il est difficile d'éviter de motiver a posteriori de tels arrêts. Il faut se garder d'attribuer à un défaut d'écriture, de structure ce qui peut-être n'était que conjoncture. Le deuxième écueil, propre au sujet même de ces séries, c'est la confusion des niveaux. Comme ce sont des séries sur des émissions, il est tentant de superposer les stratégies des scénaristes et celles des personnages dans la fiction : il y a, à chaque fois, une mise en abyme assez évidente entre les producteurs de l'émission dans la fiction et les scénaristes qui écrivent la série.

Je souhaiterais ici mesurer l'aptitude à durer de ces séries qui optent pour un propos avant tout descriptif (faire découvrir les ficelles de fabrication, les dessous et surtout les contraintes d'un programme télévisé), ce qui les prive, au fil des saisons, d'un suspense

\footnotetext{
${ }_{5}$ La réussite de Tina Fey face au géant Sorkin fut présentée comme la victoire de David contre Goliath. Forte du succès de The West Wing, Studio 60 partait favorite face à 30 Rock qui faisait figure d'outsider. Voir Erik Voss, "The Day Comedy Won: How 30 Rock Beat Studio 60 on the Sunset Strip ", Splitslider, 17 décembre 2010, http://splitsider.com/2010/12/the-day-comedy-won-a-look-back-at-studio-60-on-thesunset-strip/ (consulté le 15 juin 2015).

${ }^{6}$ Le même reproche a pu être fait à Sports Night, qui avait le format d'une sitcom. Sorkin se battit avec la production pour qu'elle renonce à lui imposer le laugh track (rire enregistré). Son écriture s'accommode en effet très mal de cette habitude de sitcom et d'ailleurs, au fil des épisodes, c'est Sorkin qui finit par l'emporter puisque les rires enregistrés tendent à s'effacer progressivement jusqu'à disparaître à la saison 2.

7 Après avoir analysé la nature du génie Zuckerberg dans The Social Network, Sorkin travaillerait actuellement au scénario d'un film sur un autre mythe moderne, Steve Jobs, adapté de la biographie de Walter Isaacson, Steve Jobs : a Biography, New York, Simon \& Schuster, 2011 (disponible en français, trad. Dominique Defert et Carole Delporte, Paris, J.C. Lattès, 2011)
} 
à long terme au profit d'une tension à courte portée (les personnages parviendront-ils à boucler leur show à temps ?).

J'étudierai d'abord les trois pilotes qui lancent un arc narratif identique (la tension entre l'ambition de qualité et les impératifs économiques qui entravent les personnages dans l'accomplissement d'une émission de haut niveau) puis la manière dont chacune de ces séries organise la suite, ralentissant ou préparant sa chute. On verra qu'elles adoptent des stratégies différentes dans leur rapport au feuilleton et au cycle, dans leur mode d'insertion d'intrigues à suspense et dans leur façon de finir sans en finir.

\section{Pilotes-manifestes : recommencer en mieux}

On a pu dire que les séries de Sorkin adoptaient des partis pris anachroniques: ce dernier dénonçait le déclin de la télévision au moment précis où elle entrait dans un nouvel âge d'or, précisément grâce à l'anoblissement du genre de la série. Ce reproche va de pair avec un autre: la nostalgie de Sorkin pour des époques de grandeur américaine révolue, l'idéalisation d'un passé qui n'a jamais existé8.

Les pilotes des trois séries sont construits sur le même jeu autour du mot program: l'émission que les héros veulent bâtir est présentée comme un programme politique, une promesse faite, par le procédé de la double énonciation, aux responsables de la chaîne et aux spectateurs. Les personnages prononcent de véritables professions de foi dans des moments de forte tension dramatique où l'on met fin à une ancienne forme d'émission et à des choix éditoriaux guidés par la recherche du compromis et des audiences. Dans le pilote de The West Wing, les héros entrent tout juste dans leurs nouvelles fonctions, au début d'une présidence, après avoir gagné l'élection. Les personnages doivent passer des promesses de campagne à leur réalisation, alors que dans les trois séries qui nous intéressent, les responsables des émissions, qui n'en sont pas à leur première expérience, décident de repartir à zéro, de recommencer en mieux, de faire prendre un nouveau départ à leur show.

Sports Night s'ouvre sur une crise : Casey McCall qui présente l'émission sportive avec son partenaire Dan Rydell s'est séparé de sa femme et a quitté le domicile conjugal, ce qui affecte son professionnalisme et menace toute l'équipe. Les responsables de la chaîne proposent à Dan de trouver un autre partenaire et Casey fait part de sa volonté de démissionner, de quitter le milieu du journalisme sportif gangréné par les faits divers et la starisation des sportifs. Casey a perdu sa passion pour le sport. L'engagement d'un nouvel analyste

8 Voir Emily Nussbaum, « Broken News. The artificial intelligence of The Newsroom », The New Yorker, 25 juin 2012, http://www.newyorker.com/magazine/2012/o6/25/brokennews (consulté le 15 juin 2015). 
pour rejoindre l'équipe, Jeremy Goodwyn (Joshua Malina), un passionné qui déclare à son entretien d'embauche avoir vu toutes les émissions et s'être décidé pour ce métier en regardant ce show annonce la promesse d'un sang neuf, d'une revitalisation de l'émission. Le premier épisode est construit sur une très forte polarisation entre deux types de personnages, dans une présentation très manichéenne : d'un côté, the good guys, les passionnés de sport, mus par la poursuite de l'excellence et refusant toute compromission ; de l'autre, les suits, les responsables de la chaîne, qui craignent la censure des lobbies de contrôle et qui sont obsédés par les chiffres de l'audience. Il apparaît très clairement que la tension qui régira le programme narratif de toute la saison sera le conflit entre show et network.

Dans le pilote de Studio 6o, le producteur de l'émission Wes Mendel est harcelé par un responsable de la censure pour couper un sketch moquant les chrétiens intégristes. Il finit par accepter, mais interrompt l'émission en plein tournage pour s'adresser au public face caméra et dénoncer les travers de la télévision ${ }^{9}$. Cette crise survient précisément au moment où Jordan McDeere, la nouvelle responsable des programmes de divertissement s'apprête à entrer en fonction. Les choix sont les mêmes que dans Sports Night: ouverture sur une situation de crise, arrivée d'une personne ou d'une situation providentielle et opposition forte entre les cadres de la chaîne et les créateurs. Au début de la saison 1, Danny dit d'ailleurs à Jordan : « you look like one of them but you talk like one of us ", " vous ressemblez à l'un d'entre eux mais vous parlez comme l'une des nôtres ». Renvoyé, Wes Mendel est donc immédiatement remplacé par deux anciens producteur et auteur de l'émission, Daniel Tripp et Matthew Albie, qui ont démissionné quelques années plus tôt de l'émission justement à cause de la censure d'un sketch. Toute la saison sera tendue vers cette fin : ne pas refaire les mêmes erreurs. Les personnages luttent contre une histoire qui semble se répéter. Quelques épisodes en analepses (1. 15, 20 et 21) permettent de comparer les choix faits dans le passé et ceux faits dans le présent de la série. À mesure que la saison avance, l'histoire se récrit sous nos yeux et l'on s'apercoit que le passé n'était pas ce qu'on croyait et qu'il fallait nuancer l'opposition frontale entre les responsables de la chaîne et les créateurs de l'émission. Ainsi Jack Rudolph, le président de la chaîne aux deux époques, n'est-il pas le responsable du départ des deux héros: c'est bien leur mentor Wes Mendel qui a cessé de les soutenir. Les suits ne sont pas toujours les ennemis que le pilote montrait.

Au début de the Newsroom, Will McAvoy est présenté comme un journaliste qui ne menace personne et caresse tous les politiques

\footnotetext{
9 Peut-être un souvenir du film de Sydney Lumet, Network (1976), dans lequel un journaliste « à l'ancienne » sur le point d'être renvoyé annonce son suicide en direct, puis dénonce les travers de la télévision telle qu'elle est en train de devenir.
} 
dans le sens du poil, à tel point qu'il est surnommé « the Jay Leno of newsanchors ${ }^{10} »$. Le prégénérique du pilote s'ouvre sur une table ronde à l'université de Northwestern à laquelle participent Will, une représentante des liberals et un représentant des conservateurs. Alors que les deux figures politiques polémiquent et s'invectivent, Will, symboliquement assis au milieu entre les deux extrêmes, s'applique à rester détaché. Mais lorsqu'une étudiante lui demande ce qui fait des États-Unis le plus grand pays au monde, il craque. Croyant voir dans le public son ex-compagne et ancienne productrice de l'émission lui souffler ses répliques et alors que le modérateur insiste pour qu'il réponde honnêtement, Will finit par se lancer dans une diatribe contre ce qu'est devenue l'Amérique, comme Wes Mendel.

Plusieurs jalons décisifs sont alors posés : Will met en danger son poste de présentateur, astreint à la neutralité. Après cette sortie, quand il revient au studio, trois semaines plus tard nous indique-t-on après le générique, toute son équipe sauf une personne est partie pour produire un autre journal télévisé, diffusé plus tard. Son discours en deux parties sur le présent morose et le passé glorieux de l'Amérique, sur ce qu'elle n'est plus et ce qui permettait son âge d'or, à savoir l'information et le bon journalisme, lance un futur pour la saison : Will et sa nouvelle équipe, engagée pour changer la forme et la politique du journal télé, essaieront de recommencer à être les meilleurs. Que ce soit pour l'Amérique en général ou pour un journal télévisé en particulier, le moteur est le même : il est toujours possible - et l'on doit toujours - s'efforcer de faire mieux.

Dans les trois pilotes, l'intrigue professionnelle est inséparable de l'intrigue amoureuse: on nous signale d'emblée les anciennes histoires qui ont échoué. C'est en cela que l'arc narratif s'apparente aux intrigues des comédies du remariage : des couples ont été ensemble et ne le sont plus : Dana et Casey dans Sports night - Casey vient de divorcer, ce qui ouvre la possibilité d'une histoire avec la productrice Dana ; Matt qui reprend le show après le suicide professionnel de son mentor Wes Mendel vient de se séparer de l'une des humoristes de l'émission, Harriet Hayes; dans The Newsroom, Mackenzie, la nouvelle productrice engagée pour relancer le journal de Will est son ancienne petite amie, celle qu'il a cru voir dans le public lors de la table ronde ${ }^{11}$. C'est d'abord le fantôme de Mac qui inspire à Will son discours

\footnotetext{
10 Jay Leno est un humoriste et satiriste américain, longtemps présentateur du Tonight Show (NBC), remplacé par Jimmy Fallon en 2014. Ce surnom est donné à Will par un journaliste de Vanity Fair et cité par le modérateur de la table ronde à l'université qui explicite ainsi l'analogie : "You're popular because you don't bother anyone ". Quand il demande à Will son sentiment sur cette déclaration, ce dernier répond qu'il est jaloux de l'audimat de Jay Leno.

${ }^{11}$ Dès la fin de l'épisode pilote, le spectateur sait que Mac était bien présente dans le public, qu'il ne s'agissait pas d'une hallucination, mais elle ne le révèle à Will que dans le dernier épisode de la saison 1.
} 
incendiaire à l'université puis la vraie qui lui fait un long discours sur Don Quichotte qui doit être le modèle du reporter. La qualité du travail et des performances des héros masculins est directement liée à l'inspiration de chacune de ces femmes qu'ils cherchent à impressionner.

Après ces pilotes-manifestes, le programme est lancé sur deux fronts : l'arc narratif est construit sur une lutte entre deux impératifs : celui de la qualité et celui du succès. Comment allier la perfection - ne pas se compromettre - et les audiences ? Comment retrouver ceux qu'on a aimés et perdus en les impressionnant par la qualité de ce qu'on accomplit ? Les personnages de Sorkin dans ces trois séries sont mus par une énergie et une ambition qui reposent sur un objectif central : ne pas décevoir, ni le public ni ceux qu'on cherche à aimer. C'est d'ailleurs un thème qui prend de l'ampleur à mesure que les intrigues se développent et qui se transforme en leçon de vie : les créateurs et présentateurs d'émission ont tendance à confondre l'audience et l'amour, et à trop vouloir être aimé par des inconnus, on en perd son intégrité et donc ceux qui vous aiment et vous admirent (à moins que ce ne soit l'inverse).

Les trois épisodes pilotes sont fondés sur un pari, un acte de foi et l'avancée de la saison repose sur une question: les personnages réussiront-ils à tenir leurs engagements ou échoueront-ils à convaincre? On en conclut que la fin de la série ou du moins de la saison devra correspondre non seulement à la survie de l'émission dans la fiction, mais au maintien de son exigence d'excellence.

\section{Séries ou feuilletons : toujours recommencer?}

À la fin du pilote de Sports Night, un événement sportif vient immédiatement dénouer la crise, comme par magie : un ancien coureur sud-africain qui ne pouvait plus courir suite à une agression lors d'une manifestation participe à un marathon : Casey regardant cette course retrouve foi dans son métier et la tension est résolue dès la fin du premier épisode. Dans Studio 6o, la nouvelle émission est un premier succès et dans The Newsroom, l'arrivée d'une nouvelle équipe de jeunes journalistes enthousiastes relance Will et le convainc de se défaire progressivement de son obsession pour les taux d'audimat.

Très vite, dans ces trois séries, l'arc narratif tend à s'effacer au profit d'un modèle sériel justifié par l'arc lui-même: puisque les personnages dans le pilote veulent reconstruire, pas à pas, une émission de qualité, il semble logique que chaque épisode corresponde à l'une de ces étapes. Ainsi, chaque épisode représente la production et la réalisation d'une émission pour laquelle il y a systématiquement des obstacles particuliers à franchir. C'est petit à petit que renaît une émission et qu'elle peut progresser, se rapprocher de son idéal. 
Pour les séries télévisées, on a pu en effet distinguer deux formes majeures : le feuilleton qui suppose l'attente impatiente d'une suite et le modèle sériel pur qui accumule des épisodes construits sur le même canevas, reproductible à l'infini. Pour l'exprimer par l'exemple et même s'il n'existe pas tout à fait de modèle pur, 24 heures (Fox, 2001-2010 et 2014) est un feuilleton ; Columbo (NBC, 1968-1978 et $\mathrm{ABC}, 1989-2003)$ une série. Le feuilleton se regarde dans l'ordre, la série peut se voir dans le désordre ${ }^{12}$.

Or les trois séries fonctionnent peu ou prou sur le principe suivant : un épisode correspond à la fabrique d'une émission et tout est à recommencer à chaque fois. Dans Sports Night, chaque épisode est construit sur le même patron : réunions de la rédaction, tournage des séquences, écriture du script, etc., l'épisode s'achevant sur le début de l'émission sportive (que le spectateur ne voit quasiment jamais en entier). Studio $6 o$ figure ce recommencement permanent sous la forme d'une horloge installée dans le bureau de Matt Albie l'auteur des sketchs et réglée sur un compte à rebours jusqu'au vendredi soir, jour de l'émission. Elle repart à zéro aussitôt qu'une émission est terminée, ce qui contribue d'ailleurs à le rendre fou. Le premier épisode consacre une séquence à cette horloge et c'est sur cette dernière que se clôt le dernier épisode. La tension court d'un vendredi à l'autre et le suspense s'annule chaque fois que les héros sont parvenus à boucler leur show à temps et à en être à peu près satisfaits. Dans The Newsroom, c'est le contrat de Mac la productrice qui est sans cesse en danger : Will encore en colère depuis que Mac lui a été infidèle trois ans auparavant et désireux de la blesser a renégocié son contrat afin d'être en droit de la renvoyer à la fin de chaque semaine.

La menace de l'annulation toujours possible de l'émission tend à s'effacer au profit d'intrigues plus localisées, de contraintes à résoudre plus rapidement au sein d'un même épisode. C'est surtout Sports night qui, par son format de sitcom, ressemble le plus à une série, tandis que Studio 60 et The Newsroom ont développé des stratégies narratives qui les orientent davantage vers le feuilleton. Soit les chaînes ont poussé Sorkin à fidéliser le public en intégrant du suspense dans ses séries, condition importante pour durer, soit Sorkin a voulu lui-même éviter le caractère répétitif d'émissions s'enchaînant les unes à la suite des autres et tenter un autre modèle.

La lutte entre les cyniques et les idéalistes, entre show et network est relancée de manière cyclique par l'introduction de nouveaux personnages ou par la modification de certains rôles narratifs. Dans Sports Night et Studio 6o, Dana et Jordan, femmes puissantes entièrement dévouées à la qualité et refusant les

\footnotetext{
12 Sur cette question, voir notamment Noël Nel, «Téléfilm, feuilleton, saga, sitcom, soap opera, telenovela: quels sont les éléments clés de la sérialité ? ", CinémAction, "Les feuilletons télévisés européens ", INA, n57, octobre 1990, p. 62-66.
} 
compromissions sont menacées par des rivales arrivistes : Sally Sasser veut prendre la place de Dana et Jordan est mise en danger par l'arrivée de Hallie Galloway, directrice des programmes de téléréalité. Dans Studio 6o, les pendants négatifs de Matt sont Ricky and Ron, rivaux médiocres mis sur la touche au moment de la reprise en main du show par Matt et Danny. À chaque niveau hiérarchique, pour chaque place professionnelle, deux faces nous sont montrées, la position idéaliste et la menace cynique. Dans The Newsroom, le modèle varie : la jeune génération ambitieuse est en fait une relève. Don qui, dans le pilote, quittait l'émission de Will pour en produire une autre s'avère dans les épisodes suivants non pas une menace mais un partenaire, comme si Sorkin avait décidé d'essayer une autre recette, peut-être plus nuancée.

Le morcellement de ces séries (un épisode $=$ une émission) laisserait penser qu'elles sont potentiellement infinies. Pour finir sans en finir, deux stratégies principales sont développées : relancer la mise en feuilleton par la greffe d'une intrigue à suspense et boucler la série ou la saison sur l'indice d'une relève assurée. La mise en abyme est particulièrement frappante : il s'agit de faire réussir les personnages dans la fiction, au moment même où les scénaristes dans le réel doivent, quant à eux, faire face à la nécessité de finir. De même que dans les pilotes, il n'était pas question de commencer mais de recommencer, de même faut-il mettre fin aux séries en indiquant que les émissions, elles, pourront continuer. La fin consiste à ne pas finir dans la diégèse.

\section{Comment (ne pas) en finir ?}

Les trois pilotes partaient d'un pari : qui, de la chaîne ou des créateurs de l'émission, allait l'emporter? Or, à mesure que les saisons 1 progressaient, cet arc narratif avait tendance à s'effacer au profit de tensions locales centrées sur la production de chaque émission au jour le jour. Pourtant, le modèle sériel ne l'emporte pas tout à fait sur le modèle feuilletonesque. Il semble que ce sont surtout les fins de saisons (qui, pour Sports Night et Studio 6o, coïncident avec les fins de séries) qui se souviennent in extremis du programme lancé dans les pilotes. Les chutes obéissent à deux stratégies différentes. La première suppose de revenir au pilote en rappelant l'arc narratif de départ pour lui offrir une fin de trajectoire. La seconde consiste à réinjecter du suspense en greffant sur le canevas sériel de la réalisation d'émissions une intrigue à tension.

\subsection{La boucle : assurer la relève}

La première stratégie est perceptible à des degrés divers dans Sports Night et dans la saison 1 de The Newsroom. À la fin de la 
deuxième saison de Sports Night, on annonce que le groupe Continental Corp va vendre la chaîne et la menace de fin d'émission est de nouveau imminente. Tout le dernier épisode est construit sur l'attente de l'issue des négociations financières: qui va racheter la chaîne et l'acheteur va-t-il démanteler les programmes ? L'épisode se résout grâce à un deus ex machina, puisque l'émission est sauvée à la dernière minute par un P-DG vertueux, propriétaire de l'entreprise Quo vadimus qui rachète la chaîne en promettant de conserver Sports Night. Alors que tous les personnages se préparent à dire adieu à leur émission, à trouver un autre emploi, que Dana vit cette fin comme un échec, le mystérieux bienfaiteur dont on ignore le nom, lui certifie qu'elle n'a pas échoué et qu'elle doit se demander où elle va. Elle comprend à la fin de l'épisode qu'il s'agissait d'un indice puisque le nom de l'entreprise qui rachète signifie justement « où allons-nous ? » (Quo vadimus). La chute de la série signe la réconciliation finale de la qualité et du profit puisque Sports Night est sauvé par un millionnaire qui veut garder l'émission parce qu'elle est bonne, non parce qu'elle rapporte.

À la fin de la saison 1 de The Newsroom, Will veut tout arrêter, à cause d'un reportage très acerbe paru dans la presse qui ridiculise l'ambition de News Night. Will entendait que cet article soit un témoignage, une archive de son projet réformateur et c'est le contraire qui se produit. Le salut viendra de là où il ne l'attendait pas : la jeune étudiante qui lui demandait lors de la table ronde ce qui faisait des États-Unis le plus grand pays du monde et qu'il humiliait dans sa réponse se présente pour faire un stage et lui déclare vouloir faire partie de l'aventure et intégrer l'équipe. Ce retour d'un personnage oublié depuis le pilote permet de boucler la saison en montrant que la relève est assurée, que la jeune génération reprendra le flambeau. Will l'engage en lui disant que c'est elle qui fait de l'Amérique le plus grand pays au monde.

3.2. La relance: oublier le pilote, greffer une intrigue parallèle

À la fin de Studio 6o, l'arc narratif est définitivement oblitéré au profit d'un autre type de suspense : une intrigue nouvelle qui court sur les quatre derniers épisodes de la saison Studio 60 permet de boucler une série dont on savait qu'elle ne serait pas reconduite. Le frère d'un des acteurs qui joue les sketchs s'est engagé en Afghanistan et il est pris en otage avec deux autres volontaires. Sa capture et la demande de rançon sont annoncées par les ravisseurs tandis qu'en parallèle, la grossesse de Jordan McDeere se complique et que cette dernière doit être emmenée en urgence à l'hôpital. Ces deux lignes narratives amorcées en même temps à l'épisode 18 se dénouent dans un happy end dans le dernier épisode. Pour en finir, le scénario oublie l'intrigue 
lancée dans le pilote et concentre les enjeux narratifs uniquement sur un suspense extérieur à l'émission et sur des intrigues sentimentales, ce qu'on a pu railler en parlant du tournant « comédie romantique » de la série.

Dans la saison 2 de the Newsroom, une intrigue juridique se greffe au canevas habituel : ayant commis une faute professionnelle grave, les journalistes sont confrontés à la poursuite en justice de l'un des leurs. Le spectateur a le sentiment d'une rectification de trajectoire : la série se judiciarise pour une large part et narrativement elle se construit sur le même procédé que dans Damages (F/X, 20072010; Audience Network, 2010-2012): chaque épisode en fait découvrir un peu plus au spectateur, alors que l'idée de départ était qu'il en sache plus que les personnages : c'est ainsi que Sorkin justifiait le choix de recourir à des événements réels du passé récent, au lieu d'inventer une actualité fictive. Cette inflexion de la saison 2 permet de nuancer l'opposition qui aurait pu être caricaturale entre journalistes et responsables de la chaîne. Ces derniers peuvent se montrer tout aussi idéalistes et engagés que les journalistes qui eux-mêmes ne sont pas infaillibles. Cette greffe d'une intrigue juridique réserve au spectateur de bonnes surprises puisque le danger ne vient pas de ceux qui étaient présentés comme les ennemis à l'épisode pilote. Sorkin externalise le conflit pour le complexifier. Cette technique narrative est donc à michemin entre la greffe et la relance du pilote puisqu'il s'agit de mêler la question de la survie même du show (ses créateurs se proposant de démissionner) et la résolution d'un contentieux judiciaire.

A show like Studio 60, or The Newsroom or The West Wing or Sports night, they're a lot less forgiving than a show that begins with a dead body and ends with finding out who did it. Hopefully I'm doing better now $^{13}$

Dans cet entretien accordé au New York Times, Sorkin oppose ses propres séries aux séries policières qui ont une trajectoire prédéterminée, du meurtre à sa résolution. Façon un peu condescendante de dire que les séries qu'il crée exigent des techniques narratives autrement plus complexes pour aller du pilote à la chute. D'une certaine manière, les trois pilotes partent pourtant d'un type de tension qui s'apparente à celle des séries policières : qui, de la chaîne ou des créateurs de l'émission, l'emportera ? Il semble difficile de tenir sur la longueur avec de telles prémisses. Et en effet, soit ce nœud est ramené à l'échelle de l'épisode et non plus de la saison, dans ce cas, on renonce au feuilleton au profit d'un modèle sériel et répétitif (chaque épisode rejoue la tension du pilote mais à l'échelle locale). Soit ce nœud

13 Interview d'Aaron Sorkin, Dave Itzkoff, «Back to You: Aaron Sorkin Returns to Television With 'The Newsroom' », New York Times, 6 juin 2012, http://artsbeat.blogs.nytimes.com/2012/06/06/back-to-you-aaron-sorkin-returns-totelevision-with-the-newsroom/? php=true\& type=blogs\& $r=0$ (consulté le 15 juin 2015) 
a besoin d'être complexifié et relancé par des greffes d'intrigues au long cours. Ce qui suppose de nuancer les oppositions et de ménager des surprises (par exemple : tel personnage n'a pas la réaction que sa position dans la hiérarchie professionnelle laissait attendre).

Il me semble qu'il y a deux manières - selon qu'on est un admirateur ou un détracteur - d'appréhender ces séries sur les dessous de la télévision. Soit on voit dans la succession de ces trois séries le retour de la même formule avec variations, Sorkin réutilisant des vieilles recettes et jouant toujours avec les limites autorisées par la chaîne. Soit on décide d'y lire une volonté de toujours recommencer pour faire mieux, pour ne pas en finir, comme les personnages de la série. C'est le choix que j'ai fait car il me semble que l'utopie qu'on a voulu voir dans ces trois séries ne réside pas tout à fait où les critiques ont voulu la placer. L'utopie porte davantage sur les conditions de production et d'existence des émissions. De même qu'il n'y a pas pour Sorkin de commencement absolu, seulement des recommencements, de même n'y a-t-il pas de chute absolue, mais une relève toujours assurée. «Recommencer parce qu'on n'en a jamais fini », ce pourrait être son mot d'ordre pour l'écriture d'une série et sa définition d'une certaine idée de l'Amérique.

\section{Annexe : tableau récapitulatif}

\begin{tabular}{|c|c|c|c|}
\hline Série & $\begin{array}{l}\text { Sports Night } \\
\text { ABC, 1998-2000 } \\
2 \text { saisons, } \\
\text { 45 épisodes, } \\
22 \text { min }\end{array}$ & $\begin{array}{l}\text { Studio 6o } \\
\text { NBC, 2006-2007 } \\
\text { 1 saison, } \\
22 \text { épisodes, } \\
42 \text { min }\end{array}$ & $\begin{array}{l}\text { The Newsroom } \\
\text { HBO, 2012-2014 } \\
3 \text { saisons, } \\
25 \text { épisodes, } \\
52 \text { min. }\end{array}$ \\
\hline $\begin{array}{l}\text { Émission } \\
\text { dans la série }\end{array}$ & $\begin{array}{l}\text { Sports Night } \\
\text { CSC (cable), } \\
\text { chaîne du groupe } \\
\text { Continental Corp }\end{array}$ & $\begin{array}{l}\text { Studio 6o on the } \\
\text { Sunset Strip } \\
\text { NBS (broadcast } \\
\text { network), chaîne } \\
\text { du groupe TMG }\end{array}$ & $\begin{array}{l}\text { News Night with } \\
\text { Will McAvoy } \\
\text { ACN (cable), } \\
\text { chaîne du groupe } \\
\text { AVM }\end{array}$ \\
\hline Inspirée de & $\begin{array}{l}\text { Sports Center, } \\
\text { ESPN }\end{array}$ & $\begin{array}{l}\text { Saturday Night } \\
\text { Live, NBC }\end{array}$ & $\begin{array}{l}\text { Countdown with } \\
\text { Keith Olbermann, } \\
\text { MSNBC }\end{array}$ \\
\hline $\begin{array}{l}\text { Personnages } \\
\text { (hiérarchie } \\
\text { de la chaîne, } \\
\text { équivalence } \\
\text { des fonctions } \\
\text { et rôles } \\
\text { narratifs) }\end{array}$ & $\begin{array}{l}\text { Luther Sachs, p-dg } \\
\text { de Continental } \\
\text { Corp, représenté } \\
\text { par J.J., porte- } \\
\text { parole } \\
\text { Isaac Jaffe } \\
\text { Directeur de la } \\
\text { rédaction }\end{array}$ & $\begin{array}{l}\text { Wilson White, p-dg } \\
\text { de TMG } \\
\text { Jack Rudolph, } \\
\text { president de NBS } \\
\\
\text { Jordan McDeere } \\
\text { Directrice des } \\
\text { programmes de } \\
\text { divertissement }\end{array}$ & $\begin{array}{l}\text { Leona Lansing, p- } \\
\text { dg d'AVM } \\
\text { \& son fils Reese } \\
\text { Lansing, } \\
\text { président d'ACN } \\
\text { Charlie Skinner } \\
\text { Directeur des } \\
\text { programmes } \\
\text { d'information }\end{array}$ \\
\hline
\end{tabular}




\begin{tabular}{|c|c|c|c|}
\hline & $\begin{array}{l}\text { Dana Whitaker } \\
\text { Productrice de } \\
\text { l'émission } \\
\text { Casey McCall \& } \\
\text { Dan Rydell } \\
\text { Présentateurs. Duo } \\
\text { central (inspiré } \\
\text { des présentateurs } \\
\text { de Sports Center, } \\
\text { Keith Olbermann } \\
\text { et Dan Patrick) } \\
\text { Membres de } \\
\text { l'équipe: } \\
\text { - Nathalie Hurley, } \\
\text { productrice } \\
\text { associée senior } \\
\text { - Jeremy } \\
\text { Goodwyn, } \\
\text { producteur } \\
\text { associé, analyste } \\
\text { sportif } \\
\text { - Sam Donovan, } \\
\text { conseiller } \\
\text { audiences, etc. }\end{array}$ & $\begin{array}{l}\text { Daniel Tripp, } \\
\text { producteur de } \\
\text { l'émission \& } \\
\text { Matthew Albie, } \\
\text { headwriter } \\
\text { (Engagés pour } \\
\text { remplacer leur } \\
\text { mentor Wes } \\
\text { Mendel, renvoyé } \\
\text { dans l'épisode } \\
\text { pilote). Duo central } \\
\\
\text { Membres de } \\
\text { l'équipe : } \\
\text { The “Big Three", } \\
\text { acteurs comiques } \\
\text { et présentateurs de } \\
\text { l'émission : } \\
\text { - Harriet Hayes } \\
\text { - Simon Stiles } \\
\text { - Tom Jeter, } \\
\text { etc. }\end{array}$ & $\begin{array}{l}\text { Membres de } \\
\text { l'équipe : } \\
\text { - Jim Harper, } \\
\text { producteur } \\
\text { associé senior } \\
\text { - Maggie Jordan, } \\
\text { productrice } \\
\text { associée } \\
\text { - Don Keefer, } \\
\text { producteur du } \\
\text { journal suivant, } \\
\text { etc. }\end{array}$ \\
\hline
\end{tabular}

\section{Bibliographie}

CAVELL Stanley, Pursuits of Happiness. The Hollywood Comedy of Remarriage, Cambridge, coll. « Harvard Film Studies», Harvard University Press, 1981

ISAACSON Walter, Steve Jobs : a Biography, New York, Simon \& Schuster, 2011 (trad. Dominique Defert et Carole Delporte, Paris, J.-C. Lattès, 2011).

ITZKOFF Dave, Aaron Sorkin, « Back to You: Aaron Sorkin Returns to Television With 'The Newsroom' », New York Times, 6 juin 2012,

http://artsbeat.blogs.nytimes.com/2012/06/o6/back-to-you-aaron-sorkinreturns-to-television-with-the-newsroom/? php=true\& type=blogs \& $r=0$ (consulté le 15 juin 2015).

MCMILLAN Graeme, « Revisiting The West Wing: A Stirring, Comforting Fantasy », Time, 19 avril 2013,

http://entertainment.time.com/2013/04/19/revisiting-the-west-wing-astirring-comforting-fantasy/ (consulté le 15 juin 2015).

MEZRICH Ben, The Accidental Billionaires : The Founding Of Facebook, A Tale of Sex, Money, Genius, and Betrayal, New York, Doubleday, 2009 (trad. Lucie 
Delplanque, La Revanche d'un solitaire : la véritable histoire du fondateur de Facebook, Paris, M. Milo, 2009)

NEL Noël, «Téléfilm, feuilleton, saga, sitcom, soap opera, telenovela : quels sont les éléments clés de la sérialité ? », CinémAction, « Les feuilletons télévisés européens », INA, $\mathrm{n}^{\circ} 57$, octobre 1990, p. 62-66.

NusSBAum Emily, «Broken News. The artificial intelligence of The Newsroom ", The New Yorker, 25 juin 2012, http://www.newyorker.com/magazine/2012/o6/25/broken-news (consulté le 15 juin 2015).

RINGELBERG Kirstin, « His Girl Friday (and Every Day) : Brilliant Women Put to Poor Use » in Considering Aaron Sorkin: Essays on the Politics, Poetics ans Sleight of Hand in the Films and Televisions Series, éd. Thomas Fahy, Jefferson/Londres, McFarland and co, 2005, p. 91-100.

Voss Erik, « The Day Comedy Won: How 30 Rock Beat Studio 60 on the Sunset Strip », Splitslider, 17 décembre 2010, http://splitsider.com/2010/12/the-daycomedy-won-a-look-back-at-studio-60-on-the-sunset-strip/ (consulté le 15 juin 2015).

WAGNER Alex, Aaron SoRKIN, « Now with Alex Wagner », 28 juin 2012, MSNBC, http://www.msnbc.com/now-with-alex-wagner/watch/aaron-sorkin-coversthe-newsroom-44421187653 (consulté le 15 juin 2015)

Laure Depretto est professeur agrégé de lettres modernes et docteur en littérature française. Elle a enseigné à l'Université Paris 8 Saint-Denis, à l’Université de Rouen ainsi qu'à l'Université Paris-Est Créteil, et travaille actuellement au Service du Dictionnaire de l'Académie française comme rédactrice lexicographe. Membre de l'équipe Fabula, elle a participé aux volumes collectifs Lire contre l'auteur (P.U.V., 2012) et Théorie des textes possibles (C.R.I.N., 2012) et a codirigé, en 2014, le numéro 13 de la revue Fabula-LHT, « La bibliothèque des textes fantômes » (http://www.fabula.org/lht/13).

Laure Depretto received her Ph.D in French literature in 2012. She worked as a teaching assistant at the University Paris 8 Saint-Denis, the University of Rouen and the University Paris-Est Créteil. She is currently working for the Académie française as a lexicographer at the Service du Dictionnaire. She is a current member of Fabula and she has participated in several projects of this association, such as the volumes Lire contre l'auteur (P.U.V., 2012) and Théorie des textes possibles (C.R.I.N., 2012). Recently she coedited the $13^{\text {th }}$ issue of the on-line journal Fabula-LHT, "La bibliothèque des textes fantômes" (http://www.fabula.org/lht/13). 